\title{
MANIFESTAÇÕES CLÍNICAS OU LESÕES ASSOCIADAS A EFEITOS ADVERSOS A MEDICAMENTOS EM CAVIDADE ORAL
}

\author{
Eduardo Costa das Mercês ${ }^{1}$; Alessandra Laís Pinho Valente Pires²; Joana Dourado \\ Martins Cerqueira; Valéria Souza Freitas ${ }^{4}$.
}

1. Bolsista PIBIC/CNPq, Graduando Odontologia, Universidade Estadual de Feira de Santana, e-mail: ecmerces@gmail.com

2. Doutoranda do Programa de Pós-graduação em Saúde Coletiva, Universidade Estadual de Feira de Santana, e-mail: lecavalent@hotmail.com

3. Membro do Núcleo de Câncer Oral, Departamento de Saúde, Universidade Estadual de Feira de Santana, e-mail: martinsjoana_1@ @hotmail.com

4. Orientadora, Departamento Saúde, Universidade Estadual de Feira de Santana, e-mail: valeria.souza.freitas@gmail.com

PALAVRAS-CHAVE: Reações adversas; medicamentos; cavidade oral

\section{INTRODUÇÃOO}

A Organização Mundial de Saúde define uma reação adversa a medicamento (RAM) como uma resposta nociva a uma droga, não intencional, que ocorre com doses normalmente utilizadas no homem para a profilaxia, terapia ou diagnóstico de uma doença, ou ainda para a modificação de uma função fisiológica (FEMIANO et al., 2008). O objetivo deste trabalho foi Avaliar a prevalência de manifestações clínicas ou lesões na cavidade oral decorrentes das reações adversas a medicamentos (RAM), em indivíduos atendidos no Centro de Referência de Lesões Bucais do Núcleo de Câncer Oral (CRLB/NUCAO), no período de 2005 a 2016.

\section{MATERIAL E MÉTODOS}

Trata-se de um estudo epidemiológico observacional, descritivo, que foi conduzido por meio da revisão de prontuários odontológicos dos indivíduos portadores de lesões bucais que faziam uso de medicamentos, atendidos no CRLB/UEFS no período de 2005 a 2016 . A população do estudo foi composta 88 prontuários que atendiam aos critérios de inclusão do estudo. As variáveis descritoras estudadas incluíram; a) variáveis relacionadas a RAM (tipo de manifestação clínica ou lesão relacionada a RAM e localização anatômica); b) ao portador da RAM (idade, sexo, raça/cor, escolaridade, presença de doença sistêmica/tipo); c) as drogas relacionadas as RAM na cavidade oral (classe dos medicamentos). Os dados foram analisados utilizado o Statistical Package for the Social Sciences (SPSS), versão 17.0, submetidos à análise descritiva, utilizando para as variáveis estudadas tabelas de frequência, com suas respectivas porcentagens. O projeto de pesquisa foi aprovado no Comitê de Ética em Pesquisa da Universidade Estadual de Feira de Santana, sob o parecer $\mathrm{n}^{\circ} 114.132$ e CAAE: 5590612.7.0000.0053.

\section{RESULTADOS E DISCUSSÃO}

No presente estudo foram recrutados, inicialmente 88 casos dos prontuários odontológicos de indivíduos atendidos no Centro de Referência de Lesões Bucais do Núcleo de Câncer Oral (CRLB/NUCAO), no período de 2005 a 2016, que apresentavam algum tipo lesão oral e faziam uso de medicamentos. Do total de casos recrutados, a maioria ocorreu indivíduos no sexo feminino $(80,7 \%)$, na quinta década de vida $(35,3 \%)$, em indivíduos da cor parda $(38,6 \%)$ e com escolaridade até o nível fundamental $(46,6 \%)$ (Tabela 1$)$.

A presença de doenças sistêmicas foi observada em 52,3\% dos casos, sendo a mais frequente a hipertensão arterial $(36,4 \%)$. Do total de prontuários, em $69,3 \%$ os indivíduos relataram fazer uso de medicamentos para o controle de hipertensão arterial, exclusivo ou associado a outras classes de fármacos. 
As principais manifestações clínicas na cavidade oral das RAM são a xerostomia, alterações no paladar, lesões brancas, aumento de volume gengival ou das glândulas salivares, desordens bolhosas como o eritema multiforme, pigmentações, reações liquenóides e infecções. Além de complicações mais severas como úlceras em mucosas, angioedemas, necroses ósseas e neoplasias malignas (PORTER; SCULLY, 2000; SCULLY; BAGAN SEBASTIAN, 2004; SCULLY; VUČIĆEVIĆ BORAS et al., 2015).

No nosso estudo, a lesão mais frequentemente observada nos indivíduos que faziam uso de algum tipo de medicamento foi a hiperplasia (30,7\%). As hiperplasias gengivais relacionadas ao uso de medicamentos não são hiperplasias verdadeiras, mas aumentos gengivais associados ao uso de anticonvulsivantes, bloqueadores dos canais de cálcio, ciclosporina, eritromicina e anticoncepcionais orais (SPOLARICH, 2014; VUČIĆEVIĆ BORAS et al., 2015). Nenhuma das hiperplasias observadas no nosso estudo estava localizada em região gengival, logo tais lesões não poderiam estar associadas a RAM (Tabela 1).

TABELA 1 - Frequência (n) e percentual válido (\%) das condições de saúde e os aspectos clínicos dos indivíduos portadores de lesões orais associados ao uso de medicamentos, atendidos no CRLB - NUCAO UEFS, no período de 2005 a 2016.

\begin{tabular}{|c|c|c|}
\hline VARIÁVEIS & $\mathbf{n}$ & $\%$ \\
\hline \multicolumn{3}{|l|}{ Doença sistêmica $(\mathrm{n}=\mathbf{8 8})$} \\
\hline Sim & 46 & 52,3 \\
\hline Não & 4 & 4,5 \\
\hline Não informado & 38 & 43,2 \\
\hline \multicolumn{3}{|l|}{$\begin{array}{l}\text { Tipo de doença sistêmica } \\
(n=88)\end{array}$} \\
\hline Hipertensão & 32 & 36,4 \\
\hline Hipertensão e diabetes & 9 & 10,2 \\
\hline Diabetes & 3 & 3,4 \\
\hline Não se aplica & 44 & 50,0 \\
\hline \multicolumn{3}{|l|}{ Tipo de lesões $(n=88)$} \\
\hline Hiperplasia & 27 & 30,7 \\
\hline Mucosite ou ulceração & 26 & 29,6 \\
\hline Pigmentação & 12 & 13,6 \\
\hline Candidíase & 10 & 11,4 \\
\hline Síndrome da ardência bucal & 8 & 9,1 \\
\hline Reação liquenóide & 2 & 2,3 \\
\hline Hipossalivação ou xerostomia & 1 & 1,1 \\
\hline Eritema multiforme & 1 & 1,1 \\
\hline Fibromatose & 1 & 1,1 \\
\hline \multicolumn{3}{|l|}{$\begin{array}{l}\text { Localização das lesões } \\
(n=88)\end{array}$} \\
\hline Mucosa jugal & 26 & 29,6 \\
\hline Rebordo alveolar & 17 & 19,3 \\
\hline Palato & 24 & 27,3 \\
\hline Língua & 13 & 14,7 \\
\hline Lábio & 6 & 6,8 \\
\hline Mais de um sítio & 2 & 2,3 \\
\hline
\end{tabular}

Fonte: Própria

Um estudo transversal realizado para determinar a prevalência de lesões orais e maxilofaciais no CRLB/UEFS no período de 2000 a 2007, envolvendo uma amostra de 391 pacientes observou que a lesão mais prevalente era a hiperplasia (18.2\%), significativamente associada ao uso de próteses (SUZUKY et al., 2014).

No nosso estudo, as ulcerações e mucosites foram as segundas lesões mais frequentemente observadas $(29,6 \%)$ (Tabela 1). Os fármacos associados ao acometimento de mucosites 
severas estão nas classes do antineoplásicos. Os principais causadores são os metotrexato, 5fluorouracil, dexorrubicina e melphelan. Ulceração oral também é relatada pelo uso de imunossupressores, broncodilatadores, anticoalinérgicos, antibióticos, antiinflamatorios esteroidais (AIEs) e aintiinflamatorios não-esteroidais (AINEs) ; JINBU; DEMITSU, 2014; SPOLARICH, 2014).

As pigmentações na mucosa oral causadas por fármacos são superficiais e geralmente estão associadas o uso de antibióticos, anticoncepcionais e quimioterápicos (VUČIĆEVIĆ BORAS et al., 2015; YUAN; WOO, 2015). Na nossa amostra, 13,6\% (Tabela 1) dos indivíduos apresentavam algum tipo de pigmentação, mas apenas um caso de pigmentação, em dorso de língua, foi comprovadamente associado ao uso de quimioterápico para o tratamento de um câncer de mama.

Uma variedade de infecções oportunistas na cavidade oral, podem surgir como consequência de uma terapia imunossupressora a longo prazo. Infecções fúngicas, principalmente a candidíase, é reportada como efeito adverso secundário à terapia com antibióticos de amplo espectro ou uso de corticóides sistêmicos a longo prazo (PORTER; SCULLY, 2000; SCULLY; BAGAN SEBASTIAN, 2004, WU et al., 2010; YUAN; WOO, 2015). Dos 10 casos encontrados no nosso estudo (Tabela 1) apenas um caso de candidíase estava relacionado ao uso de corticóide sistêmico a longo prazo.

A síndrome da ardência bucal ( $\mathrm{SAB}$ ), constitui-se uma condição de etiologia multifatorial resultante de vários fatores locais, psicológicos e/ ou alterações sistêmicas, incluindo deficiências nutricionais, alterações hormonais, infecções orais, xerostomia, reações de hipersensibilidade, uso de medicamentos e doenças como o diabetes mellitus (MALTSMANTSEIKHIN et al., 2007). O uso de inibidores da enzima conversora de angiotensina tem sido relacionado a sintomas de queimação bucal e perda da sensação do paladar. Sua associação é mais fortemente reconhecida que outros medicamentos, pois, após descontinuidade da medicação, há a remissão dos sintomas (GRUSHKA et al., 2002). No nosso estudo, foram identificados 8 casos de $\mathrm{SAB}$, onde os indivíduos faziam uso de anti-hipertensivos, psicotrópicos, anti-histamínicos e AINEs. Por ser a etiologia da SAB de etiologia multifatorial, a sua associação com o uso de medicamentos deve ser vista de forma cuidadosa, considerando o seu diagnóstico diferencial com os outros fatores relacionados a ocorrência desta síndrome.

As reações liquenóides, que se apresentam comumente sob a forma de lesões brancas, são as condições inflamatórias mais comuns que afetam a mucosa oral. Muitos medicamentos como piroxicam, sulfasalazina, e glipizida têm sido relacionados ao surgimento de reações liquenóides. Inibidores de proteases do HIV, agentes anti-hipertensivos e antimaláricos, também já foram relacionados ao aparecimento dessas reações (PORTER; SCULLY, 2000; YUAN; WOO, 2015). No nosso estudo, os casos de reação liquenóide estavam relacionados a RAM em indivíduos que faziam uso de antihipertensivos.

Apenas um caso de hiposalivação foi encontrado no nosso estudo, também associado ao uso de antihipertensivo, discordando da literatura que indica que esta manifestação é a mais comumente reportada a RAM na cavidade bucal. A hiposalivação pode ser ocasionada por diversas classes de fármacos como antidepressivos, antipsicóticos, antihistamínicos, diuréticos, anti-hipertensivos, supressores do apetite, descongestionantes nasais, broncodilatadores, relaxantes musculares e antiretrovirais (MARTINS; CAÇADOR; GAETI, 2008; YUAN; WOO, 2015).

Um caso de eritema multiforme (EM) foi observado na amostra do nosso estudo. O EM é uma doença inflamatória mucocutânea, afetando basicamente o lábio e mucosa jugal. Sua etiologia não se encontra totalmente esclarecida, podendo estar associado a uma reação de hipersensibilidade a drogas, especialmente analgésicos, anticonvulsivantes, antihipertensivos e diuréticos. Seu diagnóstico é baseado na aparição repentina da lesão, associado ao uso 
anterior de um fármaco e a presença de crostas nos lábios (BASCONES-MARTÍNEZ; MUÑOZ-CORCUERA; BASCONES-ILUNDAIN, 2015; SCULLY; BAGAN SEBASTIAN, 2004; VUČIĆEVIĆ BORAS et al., 2015). O caso de EM encontrado no nosso estudo estava localizado na língua, local que não é de predileção para a RAM, e o paciente fazia uso de antihipertensivo, antiglicemiante e AINEs.

Os resultados do nosso estudo devem ser observados levando em consideração suas limitações, já que foi um estudo exploratório preliminar, que contou com uma amostra pequena, formada por conveniência, em que generalizações ou inferências limitam-se à população investigada. Dessa maneira, é recomendável a realização de novos estudos envolvendo uma maior população.

\section{CONSIDERAÇÕES FINAIS}

Os resultados do nosso estudo revelaram uma baixa prevalência das RAM na cavidade bucal dos indivíduos atendidos no Centro de Referência de Lesões Bucais do Núcleo de Câncer Oral (CRLB/NUCAO), no período de 2005 a 2016. Apesar disso, as lesões resultantes das RAM manifestaram-se com variadas apresentações clínicas, o que indica a importância do conhecimento sobre este tema pelos profissionais de saúde de modo a permitir o correto diagnóstico e tratamento destas reações.

\section{REFERÊNCIAS}

BASCONES-MARTÍNEZ, Antonio; MUÑOZ-CORCUERA, Marta; BASCONES-ILUNDAIN, Cristina. Reacciones adversas a medicamentos en la cavidad oral. Medicina Clinica v. 144, n. 3, p. 126-131 , 2015. BUDIMIR, V. et al. Oral lichen planus - retrospective study of 563 Croatian patients. Medicina Oral Patología $\begin{array}{lllllllllll}\text { Oral } & \mathbf{y} & \text { Cirugia } & \text { Bucal } & \text { v. } & 19, & \text { n. } & 3, & \text { p. } & \text { e255-e260 } & 2014 .\end{array}$ FEMIANO, F et al. Oral manifestations of adverse drug reactions: Guidelines. J Eur Acad Dermatol Venereol. v.22, p 681-91, 2008.

GRUSHKA M, EPSTEIN JB, GORSKY M. Burning mouth syndrome. Am Fam Physician, v. 65, n. 4, p. 615620 ,

JINBU, Yoshinori; DEMITSU, Toshio. Oral ulcerations due to drug medications. Japanese Dental Science $\begin{array}{llllllll}\text { Review } & \text { v. } & 50, & \text { n. } & 2, & \text { p. } & 40-46 & ,\end{array}$ MALTSMAN-TSEIKHIN A, MORICCA P, NIV D. Burning mouth syndrome: will better understanding yield better management? Pain Pract v. 7, n. $2, \quad$ p. $\quad 151-62, \quad 2007$. PORTER, S. R.; SCULLY, C. Adverse drug reactions in the mouth. Clinics in dermatology, v. 18, n. 5, p. 525532 , 2000. SCULLY, C; BAGAN SEBASTIAN, J-V. Adverse drug reactions in the orofacial region. Critical Reviews in $\begin{array}{lllllllll}\text { Oral Biology } \& \text { Medicine. } & \text { v. } & 15, & \text { n. } & 4, & \text { p. } & 221-239 & 2004 .\end{array}$ SPOLARICH, Ann Eshenaur. Risk management strategies for reducing oral adverse drug events. The journal of evidence-based dental practice v. 14 Suppl, n. June, p. 87-94.e1 , 2014.

SONG, J. E.; SIDBURY, R. An update on pediatric cutaneous drug eruptions. Clinics in dermatology, v. 32, n. 4, p. 516-523, 2014.

SUZUKI et al. Prevalence study of oral and maxillofacial lesions in a referral center in Feira de Santana, Bahia, Brazil. Universal Journal of Education and General Studies, v.3, n. 1, p. 001-008, 2014. VUČIĆEVIĆ BORAS, Vanja et al. Adverse Drug Reactions in the Oral Cavity. Acta Clin Croat v. 54, n. 54, p. 208-215

2015.

YUAN, Anna; WOO, Sook-Bin. Adverse drug events in the oral cavity. Oral surgery, oral medicine, oral pathology and oral radiology v. 119, n. 1, p. 35-47, 2015. 